\title{
Early pregnancy sex steroids and maternal risk of epithelial ovarian cancer
}

\author{
Helena Schock ${ }^{1,2}$, Heljä-Marja Surcel ${ }^{3}$, Anne Zeleniuch-Jacquotte ${ }^{4,5}$, Kjell Grankvist ${ }^{2}$, \\ Hans-Åke Lakso², Renée Turzanski Fortner', Rudolf Kaaks ${ }^{1}$, Eero Pukkala ${ }^{6,7}$, \\ Matti Lehtinen ${ }^{7}$, Paolo Toniolo ${ }^{4,8,9}$ and Eva Lundin ${ }^{2,10}$ \\ ${ }^{1}$ Division of Cancer Epidemiology, German Cancer Research Center, Im Neuenheimer Feld 280, \\ Heidelberg 69120, Germany \\ ${ }^{2}$ Department of Medical Biosciences, Umeå University, Umeå, Sweden \\ ${ }^{3}$ Unit of Sexual and Reproductive Health, National Institute for Health and Welfare, Oulu, Finland \\ ${ }^{4}$ Departments of Population Health and Environmental Medicine, New York University School of Medicine, \\ New York, New York, USA \\ ${ }^{5}$ New York University Cancer Institute, New York University School of Medicine, New York, New York, USA \\ ${ }^{6}$ Finnish Cancer Registry, Institute for Statistical and Epidemiological Cancer Research, Helsinki, Finland \\ ${ }^{7}$ School of Health Sciences, University of Tampere, Tampere, Finland \\ ${ }^{8}$ Department of Obstetrics and Gynecology, New York University School of Medicine, New York, New York, USA \\ ${ }^{9}$ Institute of Social and Preventive Medicine, Centre Hospitalier Universitaire Vaudois (CHUV), Lausanne, Switzerland \\ ${ }^{10}$ Public Health and Clinical Medicine: Nutritional Research, Umeå University, Umeå, Sweden
}

Correspondence should be addressed to $\mathrm{H}$ Schock

Email h.schock@dkfz.de

\begin{abstract}
Well-established associations between reproductive characteristics and epithelial ovarian cancer (EOC) support an involvement of sex steroid hormones in the etiology of EOC. Limited previous studies have evaluated circulating androgens and the risk of EOC, and estrogens and progesterone have been investigated in only one of the previous studies. Furthermore, there is little data on potential heterogeneity in the association between circulating hormones and EOC by histological subgroup. Therefore, we conducted a nested case-control study within the Finnish Maternity Cohort and the Northern Sweden Maternity Cohort to investigate the associations between circulating pre-diagnostic sex steroid concentrations and the histological subtypes of EOC. We identified 1052 EOC cases among cohort members diagnosed after recruitment (1975-2008) and before March 2011. Up to three controls were individually matched to each case $(n=2694)$. Testosterone, androstenedione, 17-hydroxyprogesterone (17-OHP), progesterone, estradiol $\left(E_{2}\right)$, and sex hormone-binding globulin levels were measured in serum samples collected during the last pregnancy before EOC diagnosis. We used conditional logistic regression to estimate odds ratios (ORs) and 95\% Cls. Associations between hormones and EOC differed with respect to tumor histology and invasiveness. Sex steroid concentrations were not associated with invasive serous tumors; however, doubling of testosterone and 17-OHP concentration was associated with approximately $40 \%$ increased risk of borderline serous tumors. A doubling of androgen concentrations was associated with a $50 \%$ increased risk of mucinous tumors. The risk of endometrioid tumors increased with higher $E_{2}$ concentrations (OR: 1.89 (1.20-2.98)). This large prospective study in pregnant women supports a role of sex steroid hormones in the etiology of EOC arising in the ovaries.
\end{abstract}

Key Words

- prospective study

- case-control study

- pregnancy

- sex steroids

- ovarian neoplasms http://erc.endocrinology-journals.org DOI: 10.1530/ERC-14-0282
(C) 2014 Society for Endocrinology Printed in Great Britain
Published by Bioscientifica Ltd.
Endocrine-Related Cancer

(2014) 21, 831-844 


\section{Introduction}

Worldwide, more than 200000 women are diagnosed with epithelial ovarian cancer (EOC) each year. It is the seventh most common cancer in women and the leading cause of gynecological cancer death among women with an overall 5-year survival rate of approximately $40 \%$ only in developed countries (Ferlay et al. 2014). EOC can be subdivided into invasive and borderline diseases; approximately $15 \%$ of EOC are borderline tumors with similar epidemiological risk factors to invasive tumors, but occurring at a younger age, presenting at an earlier stage, and with a more favorable prognosis (Trope et al. 2012).

Furthermore, EOC includes different histological subtypes, with the most common being serous (75\%), endometrioid (10\%), clear cell (10\%), and mucinous (3\%) (Prat 2012). Growing evidence indicates that EOC subgroups represent clinically, morphologically, and molecularly distinct diseases (Prat 2012).

EOC may develop from inclusion cysts by metaplasia of ovarian surface epithelium or implantation of other epithelia (e.g. tubal tissue; Auersperg et al. 2001, Kurman \& Shih 2010). The pathogenic mechanisms involved in the development of ovarian cancer are poorly understood. The prevailing etiological hypotheses implicate long periods of ovulatory cycles (Fathalla 1971), retrograde menstrual flow (Cramer \& Xu 1995), and endogenous, as well as exogenous, hormonal exposure (Risch 1998). Sex steroid hormones (androgens, progesterone, and estradiol $\left(\mathrm{E}_{2}\right)$ ) are likely to be involved in the etiology of EOC, as there are well-established associations between reproductive characteristics affecting sex steroid concentrations and EOC. Examples include the protective effect of pregnancy and the use of oral contraceptives (OCs), and the increased risk associated with the current use of hormone replacement therapy (Morch et al. 2009, Tsilidis et al. 2011).

To date, studies directly relating pre-diagnostic endogenous hormone concentrations to the risk of EOC are confined to androgens and results are inconclusive (Helzlsouer et al. 1995, Lukanova et al. 2003, Rinaldi et al. 2007, Tworoger et al. 2008, Ose et al. 2014). Only one small study related progesterone and $\mathrm{E}_{2}$ to the risk of EOC (Helzlsouer et al. 1995). This is most probably because concentrations of progesterone and $\mathrm{E}_{2}$ show large intraindividual variations during the menstrual cycle among premenopausal women, making measurement difficult, and concentrations in postmenopausal women are low (Rannevik et al. 1995, Speroff \& Marc 2005).

Given the sparse data on the relationship between sex steroid hormones and EOC, and the established association between parity and EOC, we investigated early pregnancy sex steroid hormones and the subsequent ovarian cancer risk in a case-control study nested in two prospective maternity cohorts. Furthermore, we present the first data, to our knowledge, on early pregnancy hormones and the risk of EOC on the basis of histological subtype and invasiveness. Androgens in early pregnancy are similar to preconception concentrations; therefore, our study characterizes premenopausal circulating androgen concentrations with a risk of EOC. On the other hand, progesterone and $\mathrm{E}_{2}$ levels are elevated in early pregnancy and their concentrations increase further during pregnancy; thus, we are able to explore whether these hormones contribute to the protective effect conferred by pregnancy.

\section{Materials and methods}

\section{Study population}

A case-control study was nested within the Finnish Maternity Cohort (FMC) and the Northern Sweden Maternity Cohort (NSMC). These two bio-repositories store serum samples collected during the late weeks of the first, or early weeks of the second, trimester at -25 and $-20{ }^{\circ} \mathrm{C}$ respectively. The FMC was established in 1983 and includes approximately two million specimens from almost $99 \%$ of pregnancies in the country (http:// www.thl.fi/en/web/thlfi-en/research-and-expertwork/ projects-and-programmes/projects/28232, accessed May 2014). The NSMC was established in 1975 and contains almost 120000 samples from pregnancies in the three northernmost counties of Sweden (Pukkala 2011).

Study subjects were selected among members from the two cohorts with: i) no history of twin or multiple pregnancies; ii) a blood sample obtained during the first trimester of a pregnancy leading to childbirth; and iii) no history of invasive cancer (except for non-melanoma skin cancer) and/or borderline ovarian cancer before blood donation.

Identification of cases Cases diagnosed with invasive or borderline EOC after blood donation were identified through linkages with the Finnish Cancer Registry (founded in 1952) and the Swedish Cancer Registry (founded in 1958). Reporting of newly diagnosed cases is mandatory in both nationwide cancer registries, leading to very high (>95\%) completeness for solid tumors (Teppo et al. 1994, Barlow et al. 2009). We identified 1105 incident EOC cases among FMC participants and

Published by Bioscientifica Ltd. 
146 incident EOC cases among NSMC members; cases were diagnosed before December 2009 and March 2011 respectively. The serum sample from the last singleton pregnancy (or the most recent available for 68 cases) resulting in delivery of a neonate before diagnosis was selected for the study.

Selection of controls Selection of controls was carried out in two steps. First, up to 12 potentially eligible controls were selected for each case through linkages with the national population registries. Controls matching the case on study cohort, age at sampling ( \pm 6 months), date of sampling ( \pm 3 months), parity at sampling $(1,2$, and $>2$ children), and parity at diagnosis (1, 2, and $>2$ children) were selected using incidence density sampling. In a second step, up to three controls were selected at random.

Cases with insufficient serum volume for laboratory measurements (FMC: $n=157$ and NSMC: $n=31$ ) and cases for whom no eligible controls could be identified (FMC: $n=5$ and NSMC: $n=6$ ) were excluded. Therefore, a total of 1052 EOC cases (FMC: $n=943$ and NSMC: $n=109$ ) and 2694 controls (FMC: $n=2383$ and NSMC: $n=311$ ) were included in the study.

Morphology codes were provided by the Finnish and Swedish cancer registries and recoded according to the World Health Organization guidelines (Lee et al. 2003) to histological subgroups of EOC as serous $(n=477,45 \%)$, mucinous $(n=356,34 \%)$, endometrioid $(n=102,10 \%)$, clear cell $(n=26,3 \%)$, and not otherwise specified (NOS) $(n=66,6 \%)$ tumors. Data on histology were not available for 25 cases (2\%), and, thus, they were included only in overall analyses and analyses of tumor invasiveness, as were tumors diagnosed as NOS. Data on the stage of EOC at diagnosis were not available for cases from the NSMC, but were available for $87 \%$ of cases from the FMC. For cases from the FMC, stage I $(n=482,58 \%)$ was classified as localized, whereas stages II $(n=6)$, III $(n=306)$, and IV $(n=30)$ were classified as advanced EOC $(n=342,42 \%)$.

Characteristics related to pregnancy (e.g. pregnancy length, smoking during pregnancy, maternal age at first birth, and number of incomplete pregnancies) and to the newborn (e.g. gender, and birth weight and length) were obtained through linkages from the country-specific birth registries. For 2200 members of the FMC $(n=629$ case-control sets, $60 \%$ ) information on malignant cancers diagnosed among first-degree relatives was obtained through linkages with the Finnish population and Cancer registries.

This study was approved by the ethical committees of the National Institute for Health and Welfare, Finland,
University of Umeå, Sweden, and German Cancer Research Center, Germany.

\section{Laboratory analyses}

All hormonal analyses were performed at the Clinical Chemistry Laboratory of Umeå University Hospital, Umeå, Sweden. Serum specimens of individually matched case and control subjects were always included in the same laboratory batch. The technicians performing the assays were blinded to the case, control, or quality control status of the specimens. In addition to routine laboratory quality controls, two pools of serum from each of the cohorts were created at the beginning of the study and three aliquots, undistinguishable from the test samples, were inserted in each laboratory run.

Concentrations of androgens (testosterone and androstenedione), 17-hydroxyprogesterone (17-OHP), progesterone, and $\mathrm{E}_{2}$ were quantified by HPLC tandem mass spectrometry on an Applied Biosystems API4000 triple-stage quadrupole mass spectrometer. Inter- and intra-run coefficient of variation (CV) values based on the blinded pooled quality controls were $<10$ and $<11 \%$, respectively, for samples from the FMC and $<11$ and $<9 \%$, respectively, for samples from the NSMC for all sex steroids.

Sex hormone-binding globulin (SHBG) was quantified by solid-phase competitive chemiluminescence assays on an Immulite 2000 Siemens analyzer. Inter- and intra-run $\mathrm{CV}$ values based on the blinded pool quality controls were $<10 \%$ in both cohorts.

\section{Statistical analysis}

All hormone values were $\log _{2}$-transformed to normalize their distributions and to allow for the estimation of the risk with a doubling of hormone concentration. Concentrations of progesterone, 17-OHP, $\mathrm{E}_{2}$, and SHBG varied linearly with the gestational age $(r=0.49,-0.26,0.69$, and 0.57 , respectively; $P<0.0001$ ); thus, analyses including these hormones were limited to women with information on gestational age ( $n=765$ case-control sets, $73 \%)$ and gestational age was included as a covariate in the statistical models. Pearson's partial correlation coefficients were used to assess correlations between individual hormone concentrations in control samples.

We used conditional logistic regression to assess differences between cases and controls and to calculate odds ratios (ORs) and the corresponding 95\% CIs. For each of the hormones, ORs were calculated for tertiles of

Published by Bioscientifica Ltd. 
hormone concentrations using the cohort-specific frequency distribution in controls. Likelihood ratio tests were used to assess linear trends across categories based on the median hormone values for the tertiles. In addition, ORs were calculated for a unit change of $\log _{2}$-transformed hormones.

The effect of potential confounders (maternal age at first birth, smoking during index pregnancy, family history of breast and/or ovarian cancers, child's sex, and birth length and weight) was evaluated. Missing values were assigned to a 'missing' category for categorical covariates, whereas, for continuous variables, missing values were assigned the individual cohort (NSMC or FMC) median value for that variable. Analyses that excluded subjects with missing values for any of these covariates were similar to those with values imputed as described. Among the available covariates, none changed point estimates by $>10 \%$. We repeated the analyses for each of the hormones mutually adjusting for the other hormones.

Stratified analyses were performed by histology, ages at sampling and diagnosis (histology-specific; below/ above median), and the time between blood donation and diagnosis (i.e., 'lag-time'; below/above median). We also stratified by the stage (localized vs advanced disease), the number of children by diagnosis date of the matched case ( $1 \mathrm{vs}>1$ ), and the age at diagnosis (age of $<51$ vs $\geq 51$ years). The cut-off for age at diagnosis was chosen as the average age at menopause is 51 years in Sweden and Finland (Thomas et al. 2001). Tests of heterogeneity between the ORs in different subgroups were based on $\chi^{2}$ statistics, calculated as the deviations of logistic regression coefficients observed in each of the subgroups, relative to the overall regression coefficient (Whitehead \& Whitehead 1991).

We conducted sensitivity analyses limited to women diagnosed at least 2 or 3 years after blood donation to assess whether the study results were influenced by the presence of undiagnosed, but hormonally active tumors. Additionally, we performed analyses limited to women with a full-term pregnancy or, for androgen analyses, with information on gestational age at blood sampling. Results adjusted for gestational age were similar to those for hormone concentrations for each study subject computed as the difference (residual) between the assay value and the estimated cohort-specific mean value determined for the day of gestation when the sample was drawn using local linear regression (Cleveland \& Loader 1996; data not shown).

All statistical tests were two-sided with a significance level of 0.05 . Statistical analyses were performed using the Statistical Analyses System (SAS), version 9.2 Software (SAS Institute, Inc., Cary, NC, USA). The figure was prepared using the R software (package 'rmeta', function 'forestplot) version 2.15.2 (R Core Team 2014).

\section{Results}

Key characteristics of the study subjects and their newborns by study cohort are presented in Table 1 . The majority of the 1052 cases $(n=943, \mathrm{FMC}$ and $n=109$, NSMC) were diagnosed with invasive EOC $(n=642,61 \%)$. Out of them, 283 (44\%) were serous, 150 (23\%) mucinous, 102 (16\%) endometrioid, and 26 (4\%) clear cell. Among the borderline tumors $(n=410,39 \%), 194(47 \%)$ were serous and 206 (50\%) mucinous. The distribution by histological subtype and invasiveness was consistent between the two cohorts. Invasive tumors were predominantly diagnosed as advanced disease (69\%), whereas almost all borderline tumors were diagnosed as localized disease (97\%). Median age at EOC diagnosis was 43.9 years and on average 12.2 years elapsed between blood donation and diagnosis among all cases.

In the FMC, maternal and child characteristics were similar for both cases and controls except for median age at first birth (26.0 vs $26.9 ; P<0.0001)$, smoking during pregnancy ( 22 vs $14 \% ; P<0.0001$ ), and family history of breast and/or ovarian cancer ( 8 vs $5 \% ; P=0.008$ ). In the NSMC, only smoking during pregnancy was markedly different between cases and controls ( 38 vs $28 \%$; $P=0.06$ ).

Cases with mucinous tumors (borderline and invasive) had the shortest lag-time between blood collection and cancer diagnosis (10.2 years) and the youngest median age at diagnosis (40.1 and 40.9 years respectively), whereas cases diagnosed with endometrioid or clear cell tumors had the longest lag-time (16.3 and 17 years respectively) and were the oldest at diagnosis (48.1 years) (Table 2).

Cases with borderline tumors were younger at first birth, those with borderline and invasive mucinous and invasive serous tumors were more likely to smoke, and those with invasive serous tumors were more likely to have a family history of breast and/or ovarian cancer relative to their matched controls (Table 2).

Case subjects from both cohorts had significantly higher geometric means of testosterone (FMC: 0.87 vs $0.79 \mathrm{ng} / \mathrm{ml} ; P<0.0001$ and NSMC: 0.83 vs $0.72 \mathrm{ng} / \mathrm{ml}$; $P=0.004$ ) and androstenedione (FMC: $1.91 \mathrm{vs} 1.73 \mathrm{ng} / \mathrm{ml}$; $P<0.0001$ and NSMC: 1.98 vs $1.71 ; P=0.007)$ relative to control subjects. In the FMC, 17-OHP levels were also higher in cases compared with controls (2.37 vs $2.24 \mathrm{ng} / \mathrm{ml} ; P=0.006$ ).

Published by Bioscientifica Ltd. 
Table 1 Distribution of characteristics of EOC cases and their matched controls, median (min and max) or $n$ (\%) from the Finnish Maternity Cohort (1983-2009) and the Northern Sweden Maternity Cohort (1975-2011) ${ }^{a}$

\begin{tabular}{|c|c|c|c|c|c|c|c|c|}
\hline \multirow[b]{2}{*}{ Characteristics } & \multirow{2}{*}{$\begin{array}{l}\text { Missing } \\
(\%)^{\mathrm{b}}\end{array}$} & \multicolumn{2}{|c|}{ Finnish Maternity Cohort } & \multirow[b]{2}{*}{$P$} & \multirow{2}{*}{$\begin{array}{l}\text { Missing } \\
(\%)^{\mathrm{b}}\end{array}$} & \multicolumn{2}{|c|}{$\begin{array}{c}\text { Northern Sweden Maternity } \\
\text { Cohort }\end{array}$} & \multirow[b]{2}{*}{$P$} \\
\hline & & Cases (943) & Controls (2383) & & & Cases (109) & Controls (311) & \\
\hline $\begin{array}{l}\text { Age at blood donation } \\
\text { (years) }\end{array}$ & - & $31.8(16.4-45.7)$ & $31.7(15.7-45.5)$ & - & - & $29.0(17.3-41.7)$ & $28.8(17.4-42.8)$ & - \\
\hline Parity at index pregnancy & - & & & - & - & & & - \\
\hline 1 child & & $231(24 \%)$ & $597(25 \%)$ & & & $59(54 \%)$ & $170(55 \%)$ & \\
\hline 2 children & & $386(41 \%)$ & $981(41 \%)$ & & & $27(25 \%)$ & $76(24 \%)$ & \\
\hline$>2$ children & & $326(35 \%)$ & $805(34 \%)$ & & & $23(21 \%)$ & $65(21 \%)$ & \\
\hline Gestational age (days) & 25 & 75 (39-142) & $73(39-142)$ & 0.50 & - & $75(38-148)$ & $73(38-148)$ & 0.16 \\
\hline Age at first birth (years) ${ }^{c}$ & - & $26.0(14.8-46.2)$ & $26.9(14.6-45.9)$ & $<0.0001$ & 15 & $24.9(16.1-40.8)$ & $24.8(16.8-40.0)$ & 0.56 \\
\hline Pregnancy length (weeks) ${ }^{c}$ & 25 & & & 0.38 & - & & & 0.12 \\
\hline$<37$ & & $35(5 \%)$ & $84(5 \%)$ & & & $1(1 \%)$ & $13(4 \%)$ & \\
\hline$\geq 37$ & & $618(95 \%)$ & $1768(95 \%)$ & & & $108(99 \%)$ & $298(96 \%)$ & \\
\hline Child weight $(g)^{c}$ & 25 & $3620(460-5100)$ & $3610(580-5260)$ & 0.50 & - & $3460(1480-4500)$ & $3500(900-5920)$ & 0.78 \\
\hline Child length $(\mathrm{cm})^{c}$ & 25 & $51(29-57)$ & $50(25-56)$ & 0.95 & - & $50(41-55)$ & $50(33-56)$ & 0.42 \\
\hline Child gender & 0.1 & & & 0.46 & - & & & 0.18 \\
\hline Male & & $481(51 \%)$ & $1255(53 \%)$ & & & $48(44 \%)$ & $160(51 \%)$ & \\
\hline Female & & $461(49 \%)$ & $1126(47 \%)$ & & & $61(56 \%)$ & $151(49 \%)$ & \\
\hline $\begin{array}{l}\text { Smoking during } \\
\text { pregnancy }^{c}\end{array}$ & 27 & $138(22 \%)$ & $245(14 \%)$ & $<0.0001$ & 2 & $41(38 \%)$ & $85(28 \%)$ & 0.06 \\
\hline $\begin{array}{l}\text { Family history of breast } \\
\text { and/or ovarian cancer }\end{array}$ & 34 & $51(8 \%)$ & $81(5 \%)$ & 0.008 & 100 & - & - & - \\
\hline Age at diagnosis (years) & - & $43.3(19.0-66.0)$ & & & - & $47.8(20.2-68.0)$ & & \\
\hline $\begin{array}{l}\text { Years between blood } \\
\text { draw and diagnosis }\end{array}$ & - & $11.5(0.1-25.6)$ & & & - & $19.5(1.3-31.7)$ & & \\
\hline Cancer type & - & & & & 23 & & & \\
\hline Borderline & & $367(39 \%)$ & & & & $43(39 \%)$ & & \\
\hline Serous & & $174(47 \%)$ & & & & $20(51 \%)$ & & \\
\hline Mucinous & & $189(52 \%)$ & & & & $17(44 \%)$ & & \\
\hline NOS & & $4(1 \%)$ & & & & $2(5 \%)$ & & \\
\hline Invasive & & $576(61 \%)$ & & & & $66(61 \%)$ & & \\
\hline Serous & & $263(46 \%)$ & & & & $20(44 \%)$ & & \\
\hline Mucinous & & $143(25 \%)$ & & & & $7(16 \%)$ & & \\
\hline Endometrioid & & $92(16 \%)$ & & & & $10(22 \%)$ & & \\
\hline Clear Cell & & $23(4 \%)$ & & & & $3(7 \%)$ & & \\
\hline NOS & & $55(9 \%)$ & & & & $5(11 \%)$ & & \\
\hline Disease spread $^{\mathrm{d}}$ & 13 & & & & 100 & - & & \\
\hline Localized (stage I) & & $482(58 \%)$ & & & & & & \\
\hline Advanced (stage II-IV) & & $342(42 \%)$ & & & & & & \\
\hline Hormones ${ }^{e, f}$ & & & & & & & & \\
\hline Testosterone (ng/ml) & 0.5 & $0.87(0.84-0.90)$ & $0.79(0.78-0.81)$ & $<0.0001$ & 1 & $0.83(0.76-0.90)$ & $0.72(0.68-0.75)$ & 0.004 \\
\hline Androstenedione (ng/ml) & 0.5 & $1.91(1.85-1.97)$ & $1.73(1.71-1.77)$ & $<0.0001$ & 1 & $1.98(1.81-2.16)$ & $1.71(1.63-1.81)$ & 0.007 \\
\hline 17-OHP (ng/ml) & 25 & $2.37(2.29-2.46)$ & $2.24(2.19-2.29)$ & 0.006 & 1 & $2.21(2.02-2.42)$ & $2.03(1.93-2.14)$ & 0.16 \\
\hline Progesterone (ng/ml) & 25 & $24.9(24.3-25.5)$ & $24.4(24.1-24.8)$ & 0.34 & 1 & $21.4(19.9-23.0)$ & $21.4(20.5-22.3)$ & 0.97 \\
\hline Estradiol (ng/ml) & 25 & $1.97(1.89-2.05)$ & $1.89(1.85-1.94)$ & 0.09 & 1 & $1.96(1.75-2.19)$ & $1.84(1.72-1.96)$ & 0.35 \\
\hline SHBG (nmol/l) & 27 & $211(205-218)$ & $205(201-209)$ & 0.11 & 5 & 122 (107-140) & $131(121-142)$ & 0.33 \\
\hline
\end{tabular}

NOS, not otherwise specified; 17-OHP, 17-hydroxyprogesterone; SHBG, sex hormone-binding globulin. ${ }^{a}$ Conditional logistic regression models were used to compare differences between cases and controls.

${ }^{b}$ Percentage of missing values, - indicates no missing values.

'Data from the Finnish Birth Registry are available since 1987

dPercentage of missing values among cases.

${ }^{\mathrm{e}}$ Geometric means and 10th-90th percentile of hormone concentrations (adjusted for study cohort and, except for androgens and gestational age).

${ }^{f}$ Conversion from $\mathrm{ng} / \mathrm{ml}$ to $\mathrm{nmol} / \mathrm{l}$ (SI units): testosterone $\times 3.467$, androstenedione $\times 3.49,17-\mathrm{OHP} \times 3.025$, progesterone $\times 3.18$, and estradiol $\times 3.671$.

Associations between early pregnancy hormones and EOC differed by tumor histology and, for the serous subtype, also by tumor invasiveness (Table 3 and Fig. 1). We did not observe any association between the evaluated hormones and the risk of invasive serous or clear cell

http://erc.endocrinology-journals.org DOI: 10.1530/ERC-14-0282
(C) 2014 Society for Endocrinology Printed in Great Britain tumors in our study population. High progesterone and SHBG were not associated with the risk of EOC overall or with the histological subgroups.

High testosterone was associated with a significantly increased risk of borderline serous tumors (3rd vs 


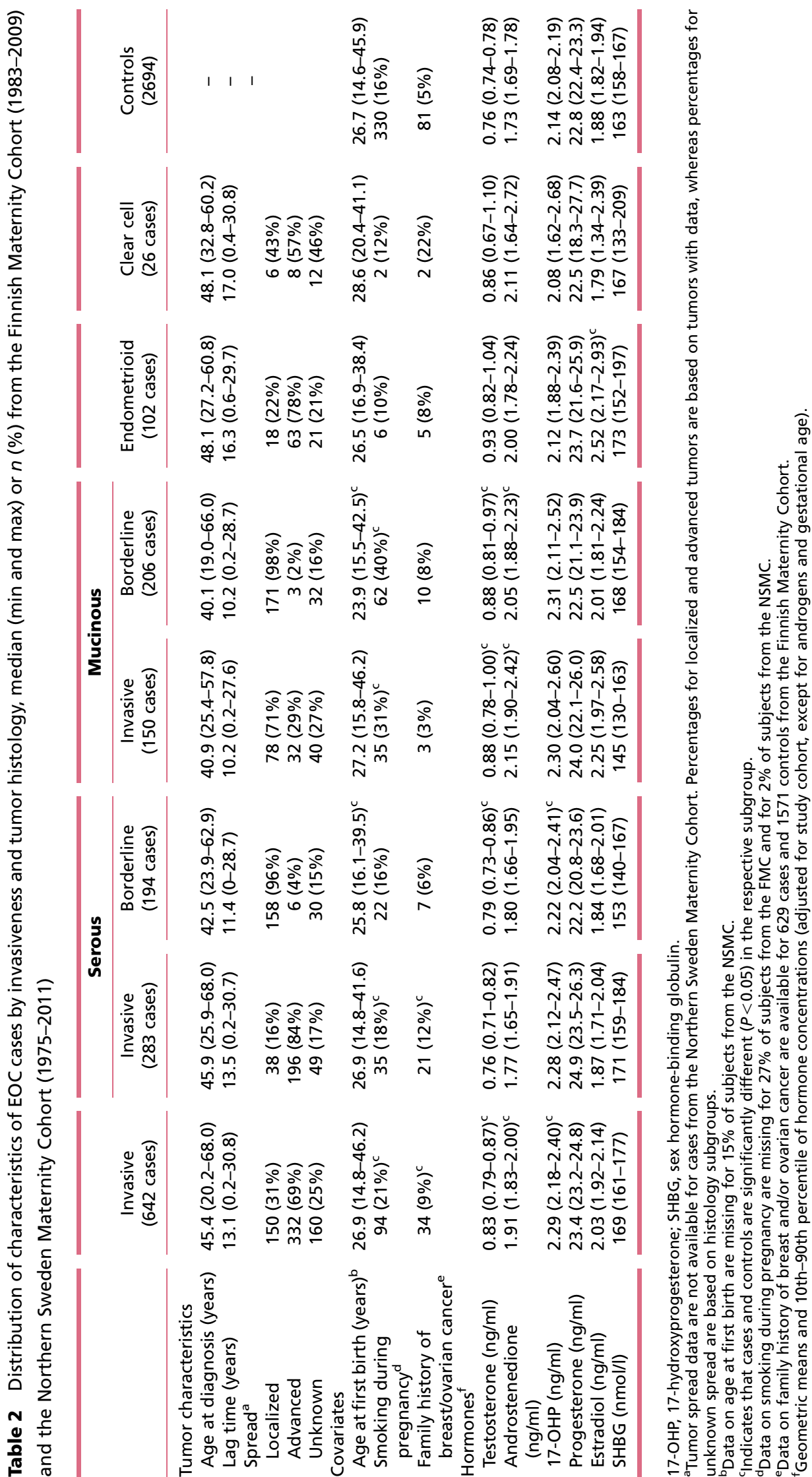


Table 3 ORs $(95 \% \mathrm{Cl})$ by tumor invasiveness and histology across tertiles of circulating hormone concentrations from the Finnish Maternity Cohort (1983-2009) and the Northern Sweden Maternity Cohort (1975-2011)

\begin{tabular}{|c|c|c|c|c|c|c|}
\hline & \multirow[b]{2}{*}{ Cases/controls } & \multicolumn{3}{|c|}{ Tertiles } & \multirow[b]{2}{*}{$P_{\text {trend }}$} & \multirow[b]{2}{*}{$P_{\text {het }}{ }^{\mathrm{b}}$} \\
\hline & & $\mathrm{T}_{1}$ & $\mathrm{~T}_{2}$ & $\mathrm{~T}_{3}$ & & \\
\hline \multicolumn{7}{|l|}{ Testosterone } \\
\hline All women & $1047 / 2673$ & Ref. & $1.27(1.06-1.53)$ & $1.56(1.30-1.87)$ & $<0.0001$ & \\
\hline Invasive & $640 / 1609$ & Ref. & $1.18(0.93-1.49)$ & $1.37(1.08-1.72)$ & 0.008 & 0.08 \\
\hline Borderline & $407 / 1064$ & Ref. & $1.45(1.07-1.96)$ & $1.94(1.43-2.63)$ & $<0.0001$ & \\
\hline Invasive serous & 282/713 & Ref. & $1.11(0.78-1.57)$ & $0.99(0.70-1.41)$ & 0.97 & 0.03 \\
\hline Borderline serous & $193 / 499$ & Ref. & $1.62(1.06-2.48)$ & $1.87(1.18-2.96)$ & 0.008 & \\
\hline Invasive mucinous & $150 / 388$ & Ref. & $1.21(0.74-1.98)$ & $1.79(1.10-2.90)$ & 0.02 & 0.76 \\
\hline Borderline mucinous & $204 / 537$ & Ref. & $1.36(0.88-2.10)$ & 1.97 (1.30-2.99) & 0.001 & \\
\hline Endometrioid & $101 / 235$ & Ref. & $1.41(0.74-2.68)$ & $1.83(0.97-3.45)$ & 0.06 & \\
\hline Clear cell & $26 / 65$ & Ref. & $0.52(0.16-1.66)$ & $0.62(0.21-1.84)$ & 0.36 & \\
\hline \multicolumn{7}{|l|}{ Androstenedione } \\
\hline All women & $1049 / 2677$ & Ref. & $1.03(0.85-1.24)$ & $1.50(1.24-1.80)$ & $<0.0001$ & \\
\hline Invasive & $640 / 1609$ & Ref. & $0.91(0.71-1.16)$ & $1.36(1.08-1.72)$ & 0.004 & 0.21 \\
\hline Borderline & $409 / 1068$ & Ref. & $1.25(0.91-1.70)$ & $1.74(1.29-2.35)$ & 0.0003 & \\
\hline Invasive serous & 282/714 & Ref. & $0.98(0.69-1.41)$ & $1.25(0.88-1.77)$ & 0.19 & 0.72 \\
\hline Borderline serous & $193 / 499$ & Ref. & $1.37(0.87-2.16)$ & $1.38(0.88-2.18)$ & 0.18 & \\
\hline Invasive mucinous & $150 / 387$ & Ref. & $0.98(0.58-1.64)$ & $1.78(1.09-2.92)$ & 0.01 & 0.73 \\
\hline Borderline mucinous & $206 / 541$ & Ref. & $1.14(0.74-1.77)$ & $2.00(1.32-3.02)$ & 0.001 & \\
\hline Endometrioid & $101 / 235$ & Ref. & $0.71(0.37-1.38)$ & $0.98(0.54-1.79)$ & 0.85 & \\
\hline Clear cell & $26 / 65$ & Ref. & $0.90(0.27-3.06)$ & $1.00(0.33-3.07)$ & 0.99 & \\
\hline \multicolumn{7}{|l|}{ 17-ОНР } \\
\hline All women & $762 / 2138$ & Ref. & $1.05(0.85-1.30)$ & $1.26(1.01-1.56)$ & 0.04 & \\
\hline Invasive & $451 / 1251$ & Ref. & $0.90(0.68-1.18)$ & $1.10(0.83-1.46)$ & 0.48 & 0.14 \\
\hline Borderline & $311 / 887$ & Ref. & $1.35(0.96-1.88)$ & $1.53(1.09-2.15)$ & 0.02 & \\
\hline Invasive serous & $198 / 555$ & Ref. & $0.96(0.63-1.44)$ & $1.09(0.72-1.67)$ & 0.69 & 0.11 \\
\hline Borderline serous & $147 / 418$ & Ref. & $1.56(0.96-2.55)$ & $1.85(1.14-2.99)$ & 0.02 & \\
\hline Invasive mucinous & $114 / 321$ & Ref. & $0.68(0.38-1.21)$ & $1.11(0.65-1.91)$ & 0.56 & 0.71 \\
\hline Borderline mucinous & $156 / 445$ & Ref. & $1.25(0.78-2.00)$ & $1.28(0.78-2.08)$ & 0.35 & \\
\hline Endometrioid & $60 / 159$ & Ref. & $1.12(0.53-2.37)$ & $0.84(0.38-1.85)$ & 0.69 & \\
\hline Clear cell & $17 / 46$ & Ref. & $0.61(0.15-2.52)$ & $0.52(0.10-2.76)$ & 0.42 & \\
\hline \multicolumn{7}{|l|}{ Progesterone } \\
\hline All women & $761 / 2136$ & Ref. & $0.88(0.71-1.08)$ & $0.96(0.76-1.21)$ & 0.67 & \\
\hline Invasive & $451 / 1252$ & Ref. & $0.90(0.68-1.19)$ & $0.99(0.73-1.35)$ & 0.95 & 0.71 \\
\hline Borderline & $310 / 884$ & Ref. & $0.85(0.62-1.18)$ & $0.91(0.63-1.31)$ & 0.56 & \\
\hline Invasive serous & $198 / 555$ & Ref. & $0.98(0.64-1.48)$ & $1.03(0.65-1.63)$ & 0.93 & 0.96 \\
\hline Borderline serous & $146 / 415$ & Ref. & $0.86(0.53-1.41)$ & $1.05(0.61-1.82)$ & 0.92 & \\
\hline Invasive mucinous & $114 / 322$ & Ref. & $1.04(0.59-1.85)$ & $1.11(0.60-2.06)$ & 0.73 & 0.47 \\
\hline Borderline mucinous & $156 / 445$ & Ref. & $0.75(0.48-1.18)$ & $0.83(0.50-1.37)$ & 0.42 & \\
\hline Endometrioid & $60 / 159$ & Ref. & $0.61(0.28-1.32)$ & $0.81(0.34-1.95)$ & 0.59 & \\
\hline Clear cell & $17 / 46$ & Ref. & $0.24(0.04-1.53)$ & $0.47(0.10-2.23)$ & 0.43 & \\
\hline \multicolumn{7}{|l|}{ Estradiol } \\
\hline All women & $761 / 2136$ & Ref. & $1.01(0.81-1.26)$ & $1.24(0.94-1.62)$ & 0.11 & \\
\hline Invasive & $451 / 1252$ & Ref. & $1.00(0.75-1.33)$ & $1.26(0.88-1.79)$ & 0.20 & 0.88 \\
\hline Borderline & $310 / 884$ & Ref. & $1.03(0.73-1.46)$ & $1.20(0.79-1.84)$ & 0.36 & \\
\hline Invasive serous & $198 / 555$ & Ref. & $0.79(0.51-1.21)$ & $0.98(0.57-1.69)$ & 0.89 & 0.78 \\
\hline Borderline serous & $146 / 415$ & Ref. & $0.82(0.50-1.35)$ & $0.87(0.46-1.64)$ & 0.64 & \\
\hline Invasive mucinous & $114 / 322$ & Ref. & $1.06(0.59-1.89)$ & $1.54(0.77-3.07)$ & 0.21 & 0.74 \\
\hline Borderline mucinous & $156 / 445$ & Ref. & $1.42(0.85-2.35)$ & $1.80(1.00-3.22)$ & 0.04 & \\
\hline Endometrioid & $60 / 159$ & Ref. & $1.41(0.59-3.41)$ & $2.76(1.04-7.33)$ & 0.03 & \\
\hline Clear cell & $17 / 46$ & Ref. & $1.93(0.46-8.08)$ & $1.58(0.26-9.64)$ & 0.53 & \\
\hline \multicolumn{7}{|l|}{ SHBG } \\
\hline All women & $735 / 2088$ & Ref. & $1.01(0.81-1.26)$ & $1.14(0.89-1.46)$ & 0.40 & \\
\hline Invasive & $429 / 1209$ & Ref. & $1.00(0.75-1.34)$ & $1.11(0.80-1.53)$ & 0.64 & 0.79 \\
\hline Borderline & $306 / 879$ & Ref. & $1.03(0.73-1.45)$ & $1.19(0.80-1.77)$ & 0.45 & \\
\hline Invasive serous & $185 / 528$ & Ref. & $1.25(0.81-1.92)$ & $1.02(0.63-1.67)$ & 0.83 & 0.90 \\
\hline Borderline serous & $143 / 413$ & Ref. & $0.94(0.58-1.54)$ & $1.08(0.59-1.96)$ & 0.85 & \\
\hline Invasive mucinous & $113 / 319$ & Ref. & $0.73(0.40-1.31)$ & $1.37(0.72-2.62)$ & 0.56 & 0.99 \\
\hline Borderline mucinous & $155 / 443$ & Ref. & $1.20(0.73-1.98)$ & $1.38(0.79-2.43)$ & 0.27 & \\
\hline Endometrioid & $55 / 150$ & Ref. & $0.88(0.37-2.10)$ & $1.29(0.51-3.26)$ & 0.63 & \\
\hline Clear cell & $15 / 43$ & Ref. & $0.91(0.20-4.24)$ & $1.25(0.24-6.53)$ & 0.86 & \\
\hline
\end{tabular}

17-OHP, 17-hydroxyprogesterone; OR, odds ratio; SHBG, sex hormone-binding globulin.

${ }^{a}$ Adjusted for gestational age (except for testosterone and androstenedione).

${ }^{b}$ Heterogeneity was tested between invasive and borderline tumors.

http://erc.endocrinology-journals.org DOI: $10.1530 /$ ERC-14-0282
(C) 2014 Society for Endocrinology Printed in Great Britain 


\begin{tabular}{|c|c|c|}
\hline \multirow{9}{*}{ 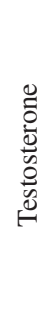 } & All women $(n=1047)$ & $1.32(1.19-1.47)$ \\
\hline & Invasive $(n=640)$ & $1.24(1.08-1.42)$ \\
\hline & Borderline $(n=407)$ & $1.47(1.23-1.76)$ \\
\hline & Invasive serous $(n=282)$ & $1.02(0.82-1.26)$ \\
\hline & Borderline serous $(n=193)$ & $1.41(1.08-1.86)$ \\
\hline & Invasive mucinous $(n=150)$ & $1.33(1.01-1.74)$ \\
\hline & Borderline mucinous $(n=204)$ & $1.50(1.18-1.90)$ \\
\hline & Endometrioid $(n=101)$ & $1.39(0.96-1.99)$ \\
\hline & Clear cell $(n=26)$ & $1.12(0.61-2.08)$ \\
\hline \multirow{9}{*}{ 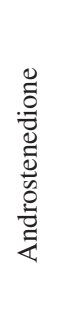 } & All women $(n=1049)$ & $1.35(1.21-1.51)$ \\
\hline & Invasive $(n=640)$ & $1.31(1.13-1.51)$ \\
\hline & Borderline $(n=409)$ & $1.43(1.20-1.72)$ \\
\hline & Invasive serous $(n=282)$ & $1.18(0.95-1.46)$ \\
\hline & Borderline serous $(n=193)$ & $1.26(0.95-1.67)$ \\
\hline & Invasive mucinous $(n=150)$ & $1.50(1.12-2.01)$ \\
\hline & Borderline mucinous $(n=206)$ & $1.56(1.22-1.99)$ \\
\hline & Endometrioid $(n=101)$ & $1.02(0.71-1.48)$ \\
\hline & Clear cell $(n=26)$ & $1.46(0.74-2.85)$ \\
\hline \multirow{9}{*}{ 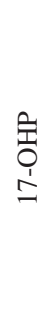 } & All women $(n=762)$ & $1.23(1.08-1.40)$ \\
\hline & Invasive $(n=451)$ & $1.21(1.02-1.43)$ \\
\hline & Borderline $(n=311)$ & $1.26(1.03-1.55)$ \\
\hline & Invasive serous $(n=198)$ & $1.16(0.90-1.51)$ \\
\hline & Borderline serous $(n=147)$ & $1.46(1.08-1.97)$ \\
\hline & Invasive mucinous $(n=114)$ & $1.30(0.93-1.82)$ \\
\hline & Borderline mucinous $(n=156)$ & $1.13(0.84-1.51)$ \\
\hline & Endometrioid $(n=60)$ & $0.90(0.54-1.49)$ \\
\hline & Clear cell $(n=17)$ & $0.66(0.26-1.65)$ \\
\hline \multirow{9}{*}{ 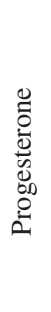 } & All women $(n=761)$ & $1.08(0.90-1.29)$ \\
\hline & Invasive $(n=451)$ & $1.12(0.89-1.42)$ \\
\hline & Borderline $(n=311)$ & $1.02(0.77-1.34)$ \\
\hline & Invasive serous $(n=198)$ & $1.18(0.83-1.66)$ \\
\hline & Borderline serous $(n=146)$ & $1.07(0.71-1.62)$ \\
\hline & Invasive mucinous ( $n=114)$ & $1.38(0.85-2.26)$ \\
\hline & Borderline mucinous $(n=156)$ & $1.04(0.70-1.53)$ \\
\hline & Endometrioid $(n=60)$ & $0.93(0.48-1.82)$ \\
\hline & Clear cell $(n=17)$ & $0.63(0.20-1.94)$ \\
\hline \multirow{9}{*}{ 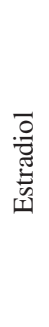 } & All women $(n=761)$ & $1.11(0.99-1.25)$ \\
\hline & Invasive $(n=451)$ & $1.11(0.95-1.28)$ \\
\hline & Borderline $(n=310)$ & $1.12(0.94-1.33)$ \\
\hline & Invasive serous $(n=198)$ & $0.93(0.74-1.17)$ \\
\hline & Borderline serous $(n=146)$ & $1.06(0.80-1.40)$ \\
\hline & Invasive mucinous ( $n=114)$ & $1.22(0.91-1.63)$ \\
\hline & Borderline mucinous $(n=156)$ & $1.24(0.98-1.57)$ \\
\hline & Endometrioid $(n=60)$ & $1.89(1.20-2.98)$ \\
\hline & Clear cell $(n=17)$ & $1.12(0.56-2.26)$ \\
\hline \multirow{9}{*}{ 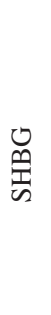 } & All women $(n=735)$ & $1.05(0.92-1.21)$ \\
\hline & Invasive $(n=429)$ & $1.04(0.87-1.24)$ \\
\hline & Borderline $(n=306)$ & $1.06(0.86-1.31)$ \\
\hline & Invasive serous $(n=185)$ & $0.98(0.74-1.29)$ \\
\hline & Borderline serous $(n=143)$ & $0.98(0.73-1.32)$ \\
\hline & Invasive mucinous $(n=113)$ & $1.09(0.77-1.56)$ \\
\hline & Borderline mucinous $(n=155)$ & $1.20(0.88-1.63)$ \\
\hline & Endometrioid $(n=55)$ & $1.10(0.64-1.89)$ \\
\hline & Clear cell $(n=15)$ & $1.11(0.39-3.16)$ \\
\hline
\end{tabular}

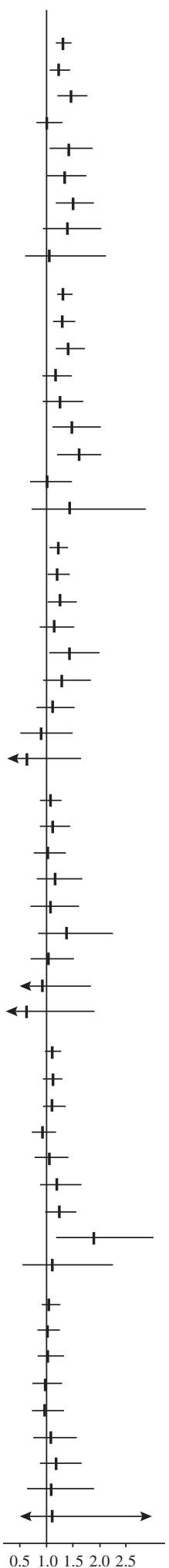

\section{Figure 1}

Risk (OR $(95 \% \mathrm{Cl})$ ) by tumor histology of epithelial ovarian cancer per unit change in $\log _{2}$ concentrations of circulating testosterone, androstenedione, 17-hydroxyprogesterone (17-OHP), progesterone, estradiol, and sex

hormone-binding globulin (SHBG), adjusted for gestational age (except for androstenedione and testosterone). The number of case-control sets is indicated in parentheses for each subgroup. http://erc.endocrinology-journals.org DOI: 10.1530/ERC-14-0282
(C) 2014 Society for Endocrinology Printed in Great Britain
Published by Bioscientifica Ltd 
1st tertile: $\left.1.87(1.18-2.96) ; P_{\text {trend }}=0.008\right)$ and an almost twofold increased risk of invasive and borderline mucinous tumors (3rd vs 1st tertile: 1.79 (1.10-2.90); $P_{\text {trend }}=0.02$ and $1.97(1.30-2.99) ; P_{\text {trend }}=0.001$ respectively). High androstenedione concentrations were associated with an increased risk of invasive and borderline mucinous tumors (3rd vs 1st tertile: 1.78 (1.09-2.92); $P_{\text {trend }}=0.01$ and $2.00(1.32-3.02) ; P_{\text {trend }}=0.001$ respectively). High concentrations of 17-OHP were associated with an increased risk of borderline serous tumors (3rd vs 1 st tertile: $\left.1.85(1.14-2.99) ; P_{\text {trend }}=0.02\right)$. A significantly increased risk was observed for high $\mathrm{E}_{2}$ concentrations for endometrioid tumors (3rd vs 1st tertile: 2.76 (1.04-7.33); $\left.P_{\text {trend }}=0.03\right)$, as well as for borderline mucinous tumors (3rd vs 1st tertile: $\left.1.80(1.00-3.22) ; P_{\text {trend }}=0.04\right)$.

We then assessed the effects of mutually adjusting for the evaluated hormones (Supplementary Table 1, see section on supplementary data given at the end of this article). Including androgens or 17-OHP in models evaluating the association of a doubling of progesterone or $\mathrm{E}_{2}$ concentrations (and vice versa) with the risk of EOC did not change the direction or the significance of the observed associations. After adjustment for androstenedione, the effect of a doubling of testosterone concentrations on invasive and borderline mucinous tumors was strongly attenuated and no longer significant (0.77 (0.43-1.36); $P=0.36$ and $1.12(0.73-1.71) ; P=0.60)$, whereas the association with endometrioid tumors was strengthened (3.45 (1.62-7.35); $P=0.001)$. A doubling of androstenedione was significantly associated with an increased risk of invasive serous tumors (1.67 (1.09-2.55); $P=0.02)$ and a decreased risk of endometrioid tumors (0.33 (0.15-0.73); $P=0.006$ ), after adjusting for testosterone. After adjustment for androgens, a doubling of 17-OHP was no longer significantly associated with the risk of borderline serous tumors (from 46 to $23 \%$ increased risk for 17-OHP).

There was no evidence of heterogeneity ( $p_{\text {het }}>0.05$ ) by age at blood donation (histology-specific; below/above median) or years between blood donation and diagnosis (histology-specific; below/above median), number of children, or study cohort. Heterogeneity by age at diagnosis ( $<51$ vs $\geq 51$ years, aproxy for menopausal status at diagnosis; Table 4 ) was observed in endometrioid tumors for a doubling of $\mathrm{E}_{2}$ concentrations ( $<51$ years: $1.36(0.84-2.22)$ and $\geq 51$ years: 14.11 (2.14-93.00); $P_{\text {het }}=0.02$ ), but as only 14 cases were diagnosed at or after the age of 51 years, this result should be regarded with caution. Although heterogeneity was not observed for invasive or invasive mucinous tumors, associations with androgens in women diagnosed at the age of $<51$ years were stronger than in older women. Heterogeneity by tumor stage at diagnosis (Table 5) was observed in invasive serous tumors for a doubling of circulating testosterone (localized: $1.91(1.02-3.57)$ and advanced: $\left.0.88(0.68-1.14) ; P_{\text {het }}=0.03\right)$ and SHBG concentrations (localized: 2.38 (1.00-5.65) and advanced: 0.88 $\left.(0.61-1.27) ; P_{\text {het }}=0.04\right)$, but not for any other hormones or EOC subtypes.

Limiting the analyses of androgens to women with information on gestational age at blood sampling did not change risk estimates with the exception of a doubling of androstenedione which was significantly associated with an increased risk of borderline serous tumors (1.50 (1.10-2.05); $P=0.01)$. Limiting the analysis to women with lag-time $>2$ or $>3$ years, or who provided a blood sample during a full-term pregnancy, did not change the risk estimates. The results did not materially change when only women who donated blood during the last pregnancy before diagnosis or selection as a control were included. Analyses limited to members of the FMC yielded similar results and analyses restricted to the NSMC ( $n=109$ cases) were in the same direction but non-significant due to the lack of power.

\section{Discussion}

This is the first prospective case-control study, to our knowledge, examining sex steroid concentrations during pregnancy and the subsequent risk of EOC. We observed heterogeneity in the associations between sex steroid hormones with respect to EOC subtypes: higher androgen concentrations were associated with an increased risk of borderline serous and mucinous tumors, whereas higher $\mathrm{E}_{2}$ was positively associated with the risk of endometrioid tumors. None of the studied hormones were associated with the risk of invasive serous tumors or clear cell tumors. Progesterone and SHBG were not associated with the risk of EOC, regardless of tumor histology and invasiveness.

There are substantial alterations in the secretion, metabolism, and concentrations of circulating hormones in maternal serum during gestation. First and early second trimester androgen concentrations are similar to those in non-pregnant women, with maternal testosterone concentration increasing gradually throughout pregnancy and androstenedione concentrations remaining relatively stable (O'Leary et al. 1991, Taylor \& Lebovic 2004). During the very first weeks of pregnancy, the corpus luteum secretes progesterone, 17-OHP, and $\mathrm{E}_{2}$ in increasing quantities. The major site of synthesis for progesterone and $E_{2}$ shifts

Published by Bioscientifica Ltd. 
Table 4 ORs $(95 \% \mathrm{Cl})$ for doubling of circulating hormone concentrations by age at diagnosis from the Finnish Maternity Cohort (1983-2009) and the Northern Sweden Maternity Cohort (1975-2011)

\begin{tabular}{|c|c|c|c|c|c|}
\hline & \multicolumn{2}{|c|}{ Age at diagnosis $<51$ years } & \multicolumn{2}{|c|}{ Age at diagnosis $\geq 51$ years } & \multirow[b]{2}{*}{$P_{\text {het }}$} \\
\hline & $\mathrm{Ca} / \mathrm{Co}$ & OR $(95 \% \mathrm{Cl})$ & $\mathrm{Ca} / \mathrm{Co}$ & OR $(95 \% \mathrm{Cl})$ & \\
\hline \multicolumn{6}{|l|}{ Invasive tumors } \\
\hline Testosterone & $475 / 1228$ & $1.32(1.12-1.55)$ & $165 / 381$ & $1.05(0.81-1.36)$ & 0.15 \\
\hline Androstenedione & $475 / 1227$ & $1.40(1.18-1.65)$ & $165 / 382$ & $1.10(0.84-1.43)$ & 0.13 \\
\hline 17-OHP & $357 / 1004$ & $1.26(1.04-1.53)$ & $94 / 247$ & $0.98(0.66-1.45)$ & 0.26 \\
\hline Progesterone & $357 / 1005$ & $1.14(0.88-1.48)$ & $94 / 247$ & $1.04(0.62-1.75)$ & 0.76 \\
\hline Estradiol & $357 / 1005$ & $1.07(0.91-1.27)$ & $94 / 247$ & $1.23(0.88-1.72)$ & 0.47 \\
\hline SHBG & $350 / 993$ & $1.04(0.85-1.27)$ & $79 / 216$ & $1.07(0.73-1.58)$ & 0.88 \\
\hline \multicolumn{6}{|c|}{ Invasive serous tumors } \\
\hline Testosterone & $195 / 506$ & $1.01(0.78-1.31)$ & $87 / 207$ & $1.04(0.72-1.53)$ & 0.88 \\
\hline Androstenedione & $195 / 506$ & $1.23(0.94-1.61)$ & $87 / 208$ & $1.10(0.77-1.57)$ & 0.63 \\
\hline 17-OHP & $145 / 413$ & $1.27(0.93-1.73)$ & $53 / 142$ & $0.91(0.55-1.51)$ & 0.27 \\
\hline Progesterone & $145 / 413$ & $1.25(0.82-1.88)$ & $53 / 142$ & $1.01(0.53-1.92)$ & 0.59 \\
\hline Estradiol & $145 / 413$ & $0.91(0.69-1.21)$ & $53 / 142$ & $0.95(0.64-1.42)$ & 0.86 \\
\hline SHBG & $142 / 407$ & $0.98(0.71-1.35)$ & $43 / 121$ & $0.98(0.53-1.78)$ & 0.98 \\
\hline \multicolumn{6}{|c|}{ Invasive mucinous tumors } \\
\hline Testosterone & $128 / 339$ & $1.46(1.07-1.99)$ & $22 / 49$ & $0.94(0.52-1.68)$ & 0.19 \\
\hline Androstenedione & $128 / 338$ & $1.64(1.18-2.28)$ & $22 / 49$ & $1.05(0.55-2.02)$ & 0.23 \\
\hline 17-OHP & $103 / 291$ & $1.29(0.90-1.84)$ & $11 / 30$ & $1.33(0.49-3.64)$ & 0.95 \\
\hline Progesterone & $103 / 292$ & $1.38(0.82-2.31)$ & $11 / 30$ & $1.19(0.24-5.78)$ & 0.86 \\
\hline Estradiol & $103 / 292$ & $1.18(0.87-1.59)$ & $11 / 30$ & $2.07(0.47-9.16)$ & 0.46 \\
\hline SHBG & $102 / 290$ & $1.10(0.72-1.67)$ & $11 / 29$ & $1.11(0.56-2.21)$ & 0.97 \\
\hline \multicolumn{6}{|c|}{ Endometrioid tumors } \\
\hline Testosterone & $67 / 159$ & $1.47(0.95-2.27)$ & $34 / 76$ & $1.20(0.62-2.32)$ & 0.61 \\
\hline Androstenedione & $67 / 159$ & $1.06(0.69-1.64)$ & $34 / 76$ & $0.92(0.45-1.90)$ & 0.74 \\
\hline 17-OHP & $41 / 111$ & $0.87(0.49-1.56)$ & $19 / 48$ & $1.00(0.36-2.76)$ & 0.82 \\
\hline Progesterone & $41 / 111$ & $0.86(0.39-1.88)$ & $19 / 48$ & $1.17(0.30-4.52)$ & 0.69 \\
\hline Estradiol & $41 / 111$ & $1.36(0.84-2.22)$ & $19 / 48$ & $14.11(2.14-93.00)$ & 0.02 \\
\hline SHBG & $39 / 108$ & $0.92(0.49-1.73)$ & $16 / 42$ & $1.84(0.61-5.56)$ & 0.28 \\
\hline
\end{tabular}

17-OHP, 17-hydroxyprogesterone; Ca, number of cases; Co, number of controls; OR, odds ratio; $P_{\text {het, }}$ test for heterogeneity; SHBG, sex hormone-binding globulin.

adjusted for gestational age (except for testosterone and androstenedione).

to the placental trophoblasts as the corpus luteum declines (after the fifth week of gestation), and concentrations of these hormones increase further, whereas the concentration of 17-OHP decreases (Taylor \& Lebovic 2004).

Support for an involvement of androgens in the pathogenesis of EOC comes from results of in vitro studies demonstrating increased cell proliferation of normal ovarian surface epithelial cells after androgen administration (Syed et al. 2001, Edmondson et al. 2002) and epidemiological studies showing a protective effect of OC use (which reduces circulating androgen levels and ovarian androgen synthesis (Lukanova \& Kaaks 2005)) (Wiegratz et al. 1995). Women diagnosed with polycystic ovary syndrome, a hyper-androgenic disorder, might be at increased risk but available data are sparse and inconclusive (Schildkraut et al. 1996, Olsen et al. 2008, Bodmer et al. 2011).

However, results from the three largest prospective studies on androgens reported thus far (Rinaldi et al. 2007,
Tworoger et al. 2008) did not show any association with the risk or report any inverse association of androstenedione for invasive serous tumors (Ose et al. 2014). In the current study, we observed an increased risk with both studied androgens for borderline serous tumors and mucinous tumors. Our study differs from previous investigations of the association between androgens and EOC in two important ways. First, this study is the first, to our knowledge, with the statistical power to study the risk associations with respect to tumor invasiveness and histology. Most of the previous reports included mainly invasive serous tumors, with negligible numbers of borderline and mucinous tumors. In the current study, we were able to investigate associations with the rarer subtypes, which are usually observed at a younger age (Chen et al. 2003). Secondly, blood samples used in our study were collected exclusively from pregnant women (by definition premenopausal); androgen concentrations are higher in premenopausal women when compared with

Published by Bioscientifica Ltd. 
Table 5 ORs $(95 \% \mathrm{Cl})$ for doubling of circulating hormone concentrations in invasive tumors by tumor stage from the Finnish Maternity Cohort (1983-2009) ${ }^{a}$

\begin{tabular}{|c|c|c|c|c|c|}
\hline & \multicolumn{2}{|c|}{ Local (stage I) } & \multicolumn{2}{|c|}{ Advanced (stages II-IV) } & \multirow[b]{2}{*}{$P_{\text {het }}$} \\
\hline & $\mathrm{Ca} / \mathrm{Co}$ & OR $(95 \% \mathrm{Cl})$ & $\mathrm{Ca} / \mathrm{Co}$ & OR $(95 \% \mathrm{Cl})$ & \\
\hline \multicolumn{6}{|l|}{ Invasive tumors } \\
\hline Testosterone & $150 / 377$ & $1.44(1.10-1.90)$ & $331 / 804$ & $1.11(0.91-1.34)$ & 0.12 \\
\hline Androstenedione & $150 / 377$ & $1.44(1.09-1.90)$ & $331 / 805$ & $1.17(0.96-1.43)$ & 0.24 \\
\hline 17-OHP & $104 / 292$ & $1.15(0.82-1.61)$ & $213 / 580$ & $1.24(0.95-1.61)$ & 0.74 \\
\hline Progesterone & $104 / 292$ & $1.18(0.70-1.98)$ & $213 / 580$ & $1.27(0.90-1.81)$ & 0.81 \\
\hline Estradiol & $104 / 292$ & $1.19(0.87-1.61)$ & $213 / 580$ & $1.09(0.87-1.36)$ & 0.66 \\
\hline SHBG & $100 / 284$ & $1.37(0.90-2.08)$ & $199 / 563$ & $1.06(0.79-1.42)$ & 0.32 \\
\hline \multicolumn{6}{|c|}{ Invasive serous tumors } \\
\hline Testosterone & $38 / 96$ & $1.91(1.02-3.57)$ & $196 / 481$ & $0.88(0.68-1.14)$ & 0.03 \\
\hline Androstenedione & $38 / 96$ & $1.60(0.91-2.80)$ & $196 / 482$ & $1.09(0.84-1.40)$ & 0.22 \\
\hline 17-OHP & $23 / 67$ & $1.37(0.75-2.51)$ & $131 / 360$ & $1.28(0.90-1.81)$ & 0.84 \\
\hline Progesterone & $23 / 67$ & $1.44(0.60-3.41)$ & $131 / 360$ & $1.33(0.84-2.09)$ & 0.88 \\
\hline Estradiol & $23 / 67$ & $1.41(0.75-2.67)$ & $131 / 360$ & $0.89(0.67-1.18)$ & 0.20 \\
\hline SHBG & $23 / 67$ & $2.38(1.00-5.65)$ & $120 / 341$ & $0.88(0.61-1.27)$ & 0.04 \\
\hline \multicolumn{6}{|c|}{ Invasive mucinous tumors } \\
\hline Testosterone & 78/198 & $1.31(0.90-1.90)$ & $32 / 83$ & $1.46(0.78-2.71)$ & 0.77 \\
\hline Androstenedione & $78 / 198$ & $1.45(0.98-2.13)$ & $32 / 83$ & $1.79(0.91-3.53)$ & 0.59 \\
\hline 17-OHP & $57 / 161$ & $1.15(0.72-1.84)$ & $22 / 63$ & $1.93(0.92-4.04)$ & 0.24 \\
\hline Progesterone & $57 / 161$ & $1.73(0.79-3.81)$ & $22 / 63$ & $2.96(0.93-9.43)$ & 0.45 \\
\hline Estradiol & $57 / 161$ & $1.23(0.82-1.86)$ & $22 / 63$ & $1.42(0.65-3.09)$ & 0.75 \\
\hline SHBG & $56 / 160$ & $1.40(0.77-2.55)$ & $22 / 63$ & $1.41(0.51-3.89)$ & 0.98 \\
\hline \multicolumn{6}{|l|}{ Endometrioid tumors } \\
\hline Testosterone & $18 / 45$ & $1.12(0.49-2.56)$ & $62 / 139$ & $1.53(0.97-2.42)$ & 0.52 \\
\hline Androstenedione & $18 / 45$ & $1.05(0.45-2.46)$ & $62 / 139$ & $1.05(0.66-1.65)$ & 0.99 \\
\hline 17-OHP & $13 / 35$ & $0.87(0.32-2.34)$ & $33 / 85$ & $0.75(0.38-1.48)$ & 0.82 \\
\hline Progesterone & $13 / 35$ & $0.43(0.07-2.72)$ & $33 / 85$ & $0.86(0.37-2.04)$ & 0.51 \\
\hline Estradiol & $13 / 35$ & $0.71(0.27-1.86)$ & $33 / 85$ & $2.15(1.06-4.36)$ & 0.07 \\
\hline SHBG & $11 / 30$ & $0.65(0.20-2.07)$ & $31 / 85$ & $1.39(0.67-2.85)$ & 0.28 \\
\hline
\end{tabular}

17-OHP, 17-hydroxyprogesterone; Ca, number of cases; Co, number of controls; OR, odds ratio; $P_{\text {het, }}$ test for heterogeneity; SHBG, sex hormone-binding globulin.

Adjusted for gestational age (except for testosterone and androstenedione).

postmenopausal women. In previous prospective studies, only 20\% (Ose et al. 2014) to 42\% (Helzlsouer et al. 1995) of cases were premenopausal.

It has been proposed that EOC originates via two main pathways of carcinogenesis and can be divided into two subtypes (type I and type II tumors; Kurman \& Shih 2011, Lim \& Oliva 2013). Type I tumors include low-grade serous and endometrioid carcinoma, clear cell, mucinous, and malignant Brenner tumors that develop slowly and are often diagnosed at an early stage. These tumors are typically confined to one ovary and progress from benign, with increasing degrees of atypia, to non-invasive and then invasive tumors. Thus, borderline ovarian tumors might be the precursors of type I tumors.

Type II tumors include high-grade serous and endometrioid carcinoma, undifferentiated, malignant mixed mesodermal, and transitional cell tumors that are highly aggressive and usually present at an advanced stage. It has been suggested that type II tumors mainly originate from the epithelium outside the ovary and invade the ovary secondarily (Kurman \& Shih 2011, Lim \& Oliva 2013).

We observed significant positive associations between androgen concentrations and borderline/invasive mucinous, as well as borderline serous subtypes, indicating that androgens may be involved in the development of the slowly growing tumors in the ovaries, whereas they do not appear to influence the risk of the most aggressive cancers, which are probably of extraovarian origin. This hypothesis is also supported by the observation in our study that risk increases for a doubling of testosterone concentrations in invasive serous tumors diagnosed as localized (proxy for type I: 1.91 (1.02-3.57)), but not advanced disease (proxy for type II: $\left.0.88(0.68-1.14) ; P_{\text {het }}=0.03\right)$.

Results from experimental studies and a number of indirect observations indicate that elevated progesterone concentrations may be inversely associated with the risk of EOC. Progesterone has been shown to have a potent apoptotic effect on the surface epithelium 
(Rodriguez et al. 1998) and to induce cellular senescence of ovarian cancer cells through FOXO1 (Diep et al. 2013). Results from epidemiological studies consistently indicate an inverse association of full-term pregnancies, increasing parity, and the use of OCs with EOC (Tsilidis et al. 2011, Merritt et al. 2013). Owing to its potent apoptotic effect and elevated production during pregnancy, progesterone is the most plausible candidate to mediate a 'washout effect', i.e., elimination from the ovary of cells that have undergone malignant transformation, a hypothesis proposed to explain the greater protective effect of pregnancies completed at an older age (Adami et al. 1994). In addition, results from observational studies have indicated that incomplete pregnancies confer less protection than a pregnancy conducted to term (Whittemore et al. 1992, Riman et al. 2002) or are not associated with the risk (Risch et al. 1994).

However, we observed no association between early pregnancy progesterone and the risk of EOC. One explanation for the lack of association could be that only the very high progesterone concentrations as observed during the third trimester are etiologically important, whereas the substantially lower hormone concentrations during the first trimester are not. Results obtained using cell culture models indicate that growth inhibition and apoptosis occur only at very high concentrations $\left(\geq 10^{-6} \mathrm{M}\right.$; Edmondson et al. 2002). In addition, during multiple pregnancies, progesterone concentrations are higher compared with singleton pregnancies and women with a history of multiple births are at a reduced risk to develop non-mucinous EOC (Whiteman et al. 2000).

Our finding of a positive association between estrogen concentrations with the risk of endometrioid tumors is novel. During pregnancy, $\mathrm{E}_{2}$ is synthesized by aromatization of androgens, mainly in the placenta, with androgens derived from the ovary, the maternal adrenal, and also de novo synthesis in placental syncytiotrophoblasts (Escobar et al. 2011). Elevated estrogen concentrations are consistently associated with an increased risk of endometrial cancer in postmenopausal women (Lukanova et al. 2004, Allen et al. 2008). Furthermore, endometrial cancer and EOC share similar risk factor profiles, as well as several common genes and pathways that are involved in their molecular pathogenesis (Merritt \& Cramer 2010). It has also been reported that patients with endometriosis, which is associated with molecular aberrations that favor increased local production of $\mathrm{E}_{2}$ (Worley et al. 2013), are at an increased risk of developing endometrioid EOC (Pearce et al. 2012). Although clear cell tumors may originate in part from endometriosis, we did not find any association between $\mathrm{E}_{2}$ and clear cell tumors. This finding might be due to the small number of cases in our study $(n=26)$, but it is consistent with the hypothesis that clear cell tumors may arise from endometriosis through mechanisms independent of hormonal signaling (Conklin \& Gilks 2013).

Our study has a number of strengths. We have presented data from two unique Maternity Cohorts in Finland and Northern Sweden and have conducted the largest prospective study on pre-diagnostic sex steroids and EOC to date (1052 vs 565 cases in the largest previous study by Ose et al. (2014)) with detailed analyses by tumor histology. As this study was conducted in a pregnant population, it was easier to measure the progesterone levels, because early pregnancy concentrations do not cycle as observed in non-pregnant premenopausal women. As the population was relatively young (median age at blood draw $=31.5$ years), we had sufficient statistical power to assess invasive and borderline tumors. Case and control subjects were tightly matched for age and date at sampling, as well as parity at the index pregnancy, thus controlling for several sources of potential confounding factors (e.g. hormone concentrations differing by parity).

A major limitation of this study is the lack of information on grade and the incomplete data on stage (available for 824 (78\%) cases). However, analyses by stage for invasive serous tumors showed the expected heterogeneity. Another limitation is the lack of data on OC use before pregnancy. However, former OC use is unlikely to influence hormone concentrations during pregnancy as steroid levels suppressed during OC use return to normal levels within one cycle after cessation (Mall-Haefeli et al. 1983). Although study samples had been stored for a long time (median 21 years) at relatively high temperatures $\left(-25 /-20^{\circ} \mathrm{C}\right)$, hormone levels were uncorrelated with time in storage, as has been reported previously (Holl et al. 2008), and case and control samples were stored under the same conditions. Another limitation is that we carried out multiple statistical tests to analyze associations with histological subtypes and thus some of our findings could be due to chance.

In summary, we provide evidence that associations of steroids and the risk of EOC vary with respect to tumor histology and invasiveness as we observed i) positive associations of androgens with borderline serous, invasive, and borderline mucinous tumors; ii) positive associations of $\mathrm{E}_{2}$ with endometrioid, and to a lesser extent with borderline tumors; and iii) no associations with invasive serous tumors. These results support a role of sex steroid hormones in the etiology of EOC arising in the ovaries, but

Published by Bioscientifica Ltd. 
do not support an association between sex steroids and invasive serous tumors, which are presumed to originate in the fallopian tubes and metastasize to the ovaries. Our findings provide additional evidence that EOC is a heterogeneous disease and indicate that further research on sex steroids and the rarer histological subtypes (endometrioid, clear cell, and mucinous) are necessary.

\section{Supplementary data}

This is linked to the online version of the paper at http://dx.doi.org/10.1530/ ERC-14-0282.

\section{Declaration of interest}

The authors declare that there is no conflict of interest that could be perceived as prejudicing the impartiality of the research reported.

\section{Funding}

This work was supported by the National Cancer Institute at the National Institutes of Health (Grant R01 CA120061) and the Lion's Cancer Foundation at Umeå University, Sweden.

\section{References}

Adami HO, Hsieh CC, Lambe M, Trichopoulos D, Leon D, Persson I, Ekbom A \& Janson PO 1994 Parity, age at first childbirth, and risk of ovarian cancer. Lancet 344 1250-1254. (doi:10.1016/S0140-6736(94)90749-8)

Allen NE, Key TJ, Dossus L, Rinaldi S, Cust A, Lukanova A, Peeters PH, Onland-Moret NC, Lahmann PH, Berrino F et al. 2008 Endogenous sex hormones and endometrial cancer risk in women in the European Prospective Investigation into Cancer and Nutrition (EPIC). Endocrine-Related Cancer 15 485-497. (doi:10.1677/ERC-07-0064)

Auersperg N, Wong AS, Choi KC, Kang SK \& Leung PC 2001 Ovarian surface epithelium: biology, endocrinology, and pathology. Endocrine Reviews 22 255-288. (doi:10.1210/edrv.22.2.0422)

Barlow L, Westergren K, Holmberg L \& Talback M 2009 The completeness of the Swedish Cancer Register: a sample survey for year 1998. Acta Oncologica 48 27-33. (doi:10.1080/02841860802247664)

Bodmer M, Becker C, Meier C, Jick SS \& Meier CR 2011 Use of metformin and the risk of ovarian cancer: a case-control analysis. Gynecologic Oncology 123 200-204. (doi:10.1016/j.ygyno.2011.06.038)

Chen VW, Ruiz B, Killeen JL, Cote TR, Wu XC \& Correa CN 2003 Pathology and classification of ovarian tumors. Cancer 97 2631-2642. (doi:10.1002/cncr.11345)

Cleveland WS \& Loader C 1996 Smoothing by local regression: principles and methods. In Statistical Theory and Computational Aspects of Smoothing, pp 10-49. Eds W Haerdle \& MG Schimek. Heidelberg: Physica-Verlag.

Conklin CM \& Gilks CB 2013 Differential diagnosis and clinical relevance of ovarian carcinoma subtypes. Expert Review of Obstetrics \& Gynecology 8 67-82. (doi:10.1586/eog.12.72)

Cramer DW \& Xu H 1995 Epidemiologic evidence for uterine growth factors in the pathogenesis of ovarian cancer. Annals of Epidemiology 5 310-314. (doi:10.1016/1047-2797(94)00098-E)

Diep CH, Charles NJ, Gilks CB, Kalloger SE, Argenta PA \& Lange CA 2013 Progesterone receptors induce FOXO1-dependent senescence in ovarian cancer cells. Cell Cycle 12 1433-1449. (doi:10.4161/cc.24550)
Edmondson RJ, Monaghan JM \& Davies BR 2002 The human ovarian surface epithelium is an androgen responsive tissue. British Journal of Cancer 86 879-885. (doi:10.1038/sj.bjc.6600154)

Escobar JC, Patel SS, Beshay VE, Suzuki T \& Carr BR 2011 The human placenta expresses CYP17 and generates androgens de novo. Journal of Clinical Endocrinology and Metabolism 96 1385-1392. (doi:10.1210/jc.2010-2504)

Fathalla MF 1971 Incessant ovulation - a factor in ovarian neoplasia? Lancet 2 163. (doi:10.1016/S0140-6736(71)92335-X)

Ferlay J, Soerjomataram I, Ervik M, Dikshit R, Eser S, Mathers C, Rebelo M, Parkin DM, Forman D, Bray F 2014. Globocan 2012 v1.0, Cancer Incidence and Mortality Worldwide: IARC CancerBase No. 11.

Helzlsouer KJ, Alberg AJ, Gordon GB, Longcope C, Bush TL, Hoffman SC \& Comstock GW 1995 Serum gonadotropins and steroid hormones and the development of ovarian cancer. Journal of the American Medical Association 274 1926-1930. (doi:10.1001/jama.1995.03530240036037)

Holl K, Lundin E, Kaasila M, Grankvist K, Afanasyeva Y, Hallmans G, Lehtinen M, Pukkala E, Surcel HM, Toniolo P et al. 2008 Effect of longterm storage on hormone measurements in samples from pregnant women: the experience of the Finnish Maternity Cohort. Acta Oncologica 47 406-412. (doi:10.1080/02841860701592400)

Kurman RJ \& Shih IM 2010 The origin and pathogenesis of epithelial ovarian cancer: a proposed unifying theory. American Journal of Surgical Pathology 34 433-443. (doi:10.1097/PAS.0b013e3181cf3d79)

Kurman RJ \& Shih IM 2011 Molecular pathogenesis and extraovarian origin of epithelial ovarian cancer - shifting the paradigm. Human Pathology 42 918-931. (doi:10.1016/j.humpath.2011.03.003)

Lee KR, Tavassoli FA, Prat J, Dietel M, Gersell DJ, Karseladze AI, Hauptmann S, Rutgers J, Russel P, Buckley CH et al. 2003 Tumors of the ovary and peritoneum. In Pathology and Genetics Tumours of the Breast and Female Genital Organs, pp 113-202. Eds FA Tavassoli \& P Devilee. Lyon: IARC Press.

Lim D \& Oliva E 2013 Precursors and pathogenesis of ovarian carcinoma. Pathology 45 229-242. (doi:10.1097/PAT.0b013e32835f2264)

Lukanova A \& Kaaks R 2005 Endogenous hormones and ovarian cancer: epidemiology and current hypotheses. Cancer Epidemiology, Biomarkers \& Prevention 14 98-107.

Lukanova A, Lundin E, Akhmedkhanov A, Micheli A, Rinaldi S, ZeleniuchJacquotte A, Lenner P, Muti P, Biessy C, Krogh V et al. 2003 Circulating levels of sex steroid hormones and risk of ovarian cancer. International Journal of Cancer 104 636-642. (doi:10.1002/ijc.10990)

Lukanova A, Lundin E, Micheli A, Arslan A, Ferrari P, Rinaldi S, Krogh V, Lenner P, Shore RE, Biessy C et al. 2004 Circulating levels of sex steroid hormones and risk of endometrial cancer in postmenopausal women. International Journal of Cancer 108 425-432. (doi:10.1002/ijc.11529)

Mall-Haefeli M, Werner-Zodrow I, Huber PR, Darragh A \& Lambe R 1983 Effects of various combined oral contraceptives on sex steroids, gonadotropins and SHBG. Irish Medical Journal 76 266-272.

Merritt MA \& Cramer DW 2010 Molecular pathogenesis of endometrial and ovarian cancer. Cancer Biomarkers 9 287-305. (doi:10.3233/CBM2011-0167)

Merritt MA, De Pari M, Vitonis AF, Titus LJ, Cramer DW \& Terry KL 2013 Reproductive characteristics in relation to ovarian cancer risk by histologic pathways. Human Reproduction 28 1406-1417. (doi:10.1093/ humrep/des466)

Morch LS, Lokkegaard E, Andreasen AH, Kruger-Kjaer S \& Lidegaard O 2009 Hormone therapy and ovarian cancer. Journal of the American Medical Association 302 298-305. (doi:10.1001/jama.2009.1052)

O'Leary P, Boyne P, Flett P, Beilby J \& James I 1991 Longitudinal assessment of changes in reproductive hormones during normal pregnancy. Clinical Chemistry 37 667-672.

Olsen CM, Green AC, Nagle CM, Jordan SJ, Whiteman DC, Bain CJ, Webb PM \& Australian Cancer Study G \& the Australian Ovarian Cancer Study G 2008 Epithelial ovarian cancer: testing the 'androgens hypothesis'. Endocrine-Related Cancer 15 1061-1068. (doi:10.1677/ ERC-08-0075) 
Ose J, Fortner RT, Rinaldi S, Schock H, Overvad K, Tjonneland A, Hansen L, Dossus L, Fournier A, Baglietto L et al. 2014 Endogenous androgens and risk of epithelial invasive ovarian cancer by tumor characteristics in the European Prospective Investigation into Cancer and Nutrition. International Journal of Cancer [in press]. (doi:10.1002/ijc.29000)

Pearce CL, Templeman C, Rossing MA, Lee A, Near AM, Webb PM, Nagle CM, Doherty JA, Cushing-Haugen KL, Wicklund KG et al. 2012 Association between endometriosis and risk of histological subtypes of ovarian cancer: a pooled analysis of case-control studies. Lancet Oncology $\mathbf{1 3}$ 385-394. (doi:10.1016/S1470-2045(11)70404-1)

Prat J 2012 Ovarian carcinomas: five distinct diseases with different origins, genetic alterations, and clinicopathological features. Virchows Archiv 460 237-249. (doi:10.1007/s00428-012-1203-5)

Pukkala E 2011 Nordic biological specimen bank cohorts as basis for studies of cancer causes and control: quality control tools for study cohorts with more than two million sample donors and 130,000 prospective cancers. In Methods in Biobanking, pp 61-112. Ed J Dillner. New York, NY: Humana Press.

R Core Team 2014 Team R: A Language and Environment for Statistical Computing. Vienna: R Foundation for Statistical Computing.

Rannevik G, Jeppsson S, Johnell O, Bjerre B, Laurell-Borulf Y \& Svanberg L 1995 A longitudinal study of the perimenopausal transition: altered profiles of steroid and pituitary hormones, SHBG and bone mineral density. Maturitas 21 103-113. (doi:10.1016/0378-5122(94)00869-9)

Riman T, Dickman PW, Nilsson S, Correia N, Nordlinder H, Magnusson CM \& Persson IR 2002 Risk factors for invasive epithelial ovarian cancer: results from a Swedish case-control study. American Journal of Epidemiology 156 363-373. (doi:10.1093/aje/kwf048)

Rinaldi S, Dossus L, Lukanova A, Peeters PH, Allen NE, Key T, Bingham S, Khaw KT, Trichopoulos D, Trichopoulou A et al. 2007 Endogenous androgens and risk of epithelial ovarian cancer: results from the European Prospective Investigation into Cancer and Nutrition (EPIC). Cancer Epidemiology, Biomarkers \& Prevention 16 23-29. (doi:10.1158/ 1055-9965.EPI-06-0755)

Risch HA 1998 Hormonal etiology of epithelial ovarian cancer, with a hypothesis concerning the role of androgens and progesterone. Journal of the National Cancer Institute 90 1774-1786. (doi:10.1093/jnci/90.23.1774)

Risch HA, Marrett LD \& Howe GR 1994 Parity, contraception, infertility, and the risk of epithelial ovarian cancer. American Journal of Epidemiology 140 585-597.

Rodriguez GC, Walmer DK, Cline M, Krigman H, Lessey BA, Whitaker RS, Dodge R \& Hughes CL 1998 Effect of progestin on the ovarian epithelium of macaques: cancer prevention through apoptosis? Journal of the Society for Gynecologic Investigation 5 271-276. (doi:10.1016/S1071-5576(98)00017-3)

Schildkraut JM, Schwingl PJ, Bastos E, Evanoff A \& Hughes C 1996 Epithelial ovarian cancer risk among women with polycystic ovary syndrome. Obstetrics and Gynecology 88 554-559. (doi:10.1016/ 0029-7844(96)00226-8)
Speroff L \& Marc AF 2005 Regulation of the menstrual cycle. In Clinical Gynecologic Endocrinology and Infertility, pp 187-231. Eds L Speroff \& AF Marc. Baltimore, MD: Lippincott Williams \& Wilkins.

Syed V, Ulinski G, Mok SC, Yiu GK \& Ho SM 2001 Expression of gonadotropin receptor and growth responses to key reproductive hormones in normal and malignant human ovarian surface epithelial cells. Cancer Research 61 6768-6776.

Taylor RN \& Lebovic DI 2004 The endocrinology of pregnancy. In Basic \& Clinical Endocrinology, pp 637-657. Eds FS Greenspan \& DG Gardner. New York, NY: Lange Medical Books/McGraw-Hill.

Teppo L, Pukkala E \& Lehtonen M 1994 Data quality and quality control of a population-based cancer registry. Experience in Finland. Acta Oncologica 33 365-369. (doi:10.3109/02841869409098430)

Thomas F, Renaud F, Benefice E, de Meeus T \& Guegan JF 2001 International variability of ages at menarche and menopause: patterns and main determinants. Human Biology 73 271-290. (doi:10.1353/ hub.2001.0029)

Trope CG, Kaern J \& Davidson B 2012 Borderline ovarian tumours. Best Practice \& Research. Clinical Obstetrics \& Gynaecology 26 325-336. (doi:10.1016/j.bpobgyn.2011.12.006)

Tsilidis KK, Allen NE, Key TJ, Dossus L, Lukanova A, Bakken K, Lund E, Fournier A, Overvad K, Hansen L et al. 2011 Oral contraceptive use and reproductive factors and risk of ovarian cancer in the European Prospective Investigation into Cancer and Nutrition. British Journal of Cancer 105 1436-1442. (doi:10.1038/bjc.2011.371)

Tworoger SS, Lee IM, Buring JE \& Hankinson SE 2008 Plasma androgen concentrations and risk of incident ovarian cancer. American Journal of Epidemiology 167 211-218. (doi:10.1093/aje/kwm278)

Whitehead A \& Whitehead J 1991 A general parametric approach to the meta-analysis of randomized clinical trials. Statistics in Medicine 10 1665-1677. (doi:10.1002/sim.4780101105)

Whiteman DC, Murphy MF, Cook LS, Cramer DW, Hartge P, Marchbanks PA, Nasca PC, Ness RB, Purdie DM \& Risch HA 2000 Multiple births and risk of epithelial ovarian cancer. Journal of the National Cancer Institute 92 1172-1177. (doi:10.1093/jnci/92. 14.1172)

Whittemore AS, Harris R \& Itnyre J 1992 Characteristics relating to ovarian cancer risk: collaborative analysis of 12 US case-control studies. II. Invasive epithelial ovarian cancers in white women. Collaborative Ovarian Cancer Group. American Journal of Epidemiology 136 1184-1203.

Wiegratz I, Jung-Hoffmann C \& Kuhl H 1995 Effect of two oral contraceptives containing ethinylestradiol and gestodene or norgestimate upon androgen parameters and serum binding proteins. Contraception 51 341-346. (doi:10.1016/0010-7824(95)00098-U)

Worley MJ, Welch WR, Berkowitz RS \& Ng SW 2013 Endometriosisassociated ovarian cancer: a review of pathogenesis. International Journal of Molecular Sciences 14 5367-5379. (doi:10.3390/ijms14035367)

Received 25 August 2014

Accepted 1 September 2014 http://erc.endocrinology-journals.org DOI: 10.1530/ERC-14-0282
(C) 2014 Society for Endocrinology Printed in Great Britain
Published by Bioscientifica Ltd. 